\title{
A K-Band Low-Power Miniaturized Hyperthermia System
}

\author{
Dongki Kim $\cdot$ Kihyun Kim $\cdot$ Jungmin Oh $\cdot$ Youngrak Park $\cdot$ Youngwoo Kwon
}

\begin{abstract}
A K-band low-power miniaturized planar-type hyperthermia system was developed to replace massive and expensive equipment. The system consists of a VCO with a buffer amplifier, a high-power amplifier module, a 20-dB-coupled line coupler, a chip circulator and two power detectors for signal generation, amplification and power monitoring. All these components have been implemented in planar form on two module blocks. The total size of the hyperthermia system was less than $10 \times 6.5 \times 3 \mathrm{~cm}^{3}$. In order to verify the system performance, ablations were carried out on nude mice xenografted with human breast cancer. Ablation results show performance comparable to the massive components-based system. This work shows the feasibility of a low-cost miniaturized hyperthermia system for practical clinical applications.
\end{abstract}

Key words : Hyperthermia, Ablation, Bio System.

\section{Introduction}

A key concept of therapeutic hyperthermia is the difference in thermal sensitivity between cancerous tissue and normal tissue ${ }^{[1]}$. This is due to the fact that tumor cells have a disorganized and compact vascular structure and therefore have difficulty dissipating heat. As a result, hyperthermia may cause cancerous cells to undergo apoptosis in direct response to applied heat, while healthy cells can more easily maintain a normal temperature $^{[2]}$. Therefore, through the careful application of heat, cancerous tissue can be destroyed, while leaving the surrounding normal tissue undamaged.

Microwave ablation systems can be categorized into interstitial and non-invasive ones. The interstitial ablation system presents the advantage of selective heating, and with optimal design, can be minimally invasive. However, this method of cancer treatment has yet to establish clinical practicality due to difficulties such as poor power efficiency, resulting in the use of extremely high power levels, along with the lack of acceptably small applicator sizes. With regards to patient discomfort and pain, the non-invasive system seems to be the most attractive approach. However, the method is not feasible unless multiple probes are inserted into the body for focusing and temperature monitoring ${ }^{[3] \sim[7]}$. As a result, interstitial ablation is much more favorable to microwaves due to practical considerations. The existing microwave/RF ablation systems need huge power ranging from tens of watts to more than $1 \mathrm{~kW}^{[3] \sim[10]}$. Most of all, the system complexity of bulky and various equip- ment make it difficult to apply such systems in clinical treatment. The goal of this work is to develop a compact and portable interstitial hyperthermia system for low-power microwave ablation. The target is to make the hyperthermia hardware small enough to be handcarried. This is achieved by the integration of key microwave components into two separate boards without any performance degradation.

\section{Design and Fabrication}

Fig. 1 is a block diagram of the miniaturized microwave hyperthermia system. The hyperthermia frequency choice in this work can be explained with the frequency-dependent permittivity plot of Fig. 2, which shows the measured complex permittivity of fat and human breast cancer xenografted onto the nude mice at up to $40 \mathrm{GHz}^{[11],[12]}$. Also, it can be seen from Fig. 2 that the difference of the dielectric loss(imaginary part) of the cancer and the fat reach their maximum around $20 \mathrm{GHz}$. The choice of hyperthermia frequencies around $20 \mathrm{GHz}$ will result in low-power/high-efficiency heating with significantly reduced collateral damage. The target frequency was $18 \mathrm{GHz}$ in consideration of the above aspects.

The total system is divided into two modules. The first one is a high-power amplifier module(PAM) and the second one is the integrated circuits of a voltagecontrolled oscillator(VCO) with a buffer amplifier, a coupler, a circulator and two power detectors. Two-module design was required to effectively dissipate the heat

Manuscript received August 21, 2009 ; revised November 10, 2009. (ID No. 20090821-034J)

Institute of New Media and Communications(INMC), School of Electrical Engineering and Computer Science, Seoul National University, Seoul, Korea. 
Module 2

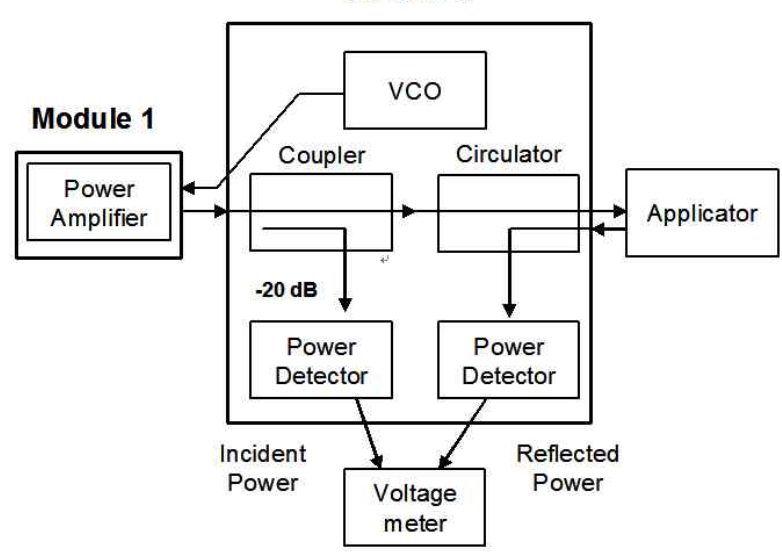

Fig. 1. A block diagram of the miniaturized microwave hyperthermia system.

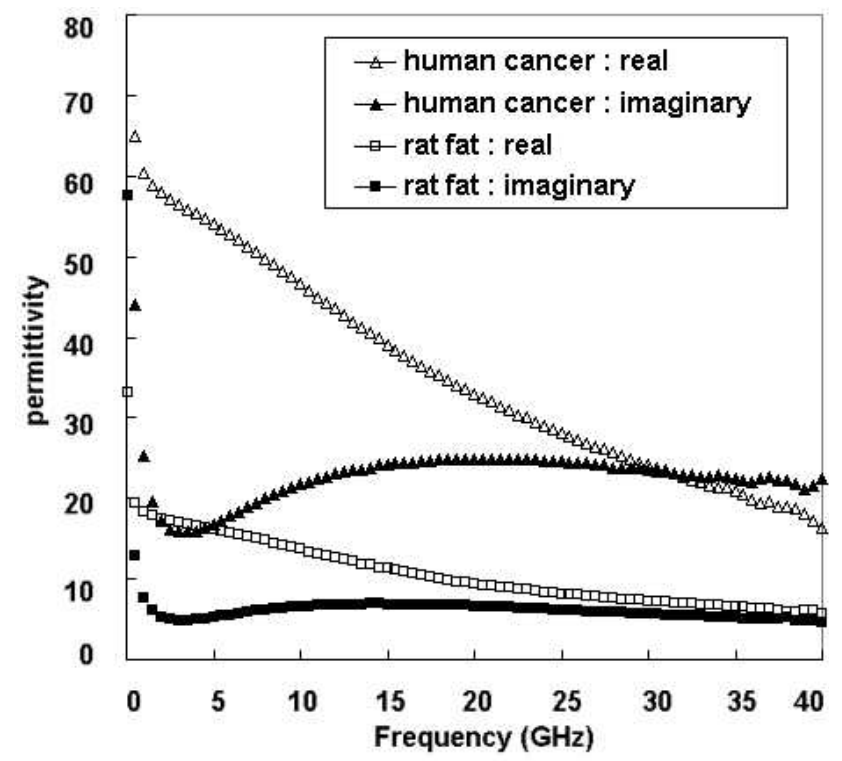

Fig. 2. Measured complex permittivities of human cancer xenografted onto the nude mice and fat extracted from rat thigh.

generated by PAM and prevent the performance degradation of modules from the high heat of the PAM. Except for the high-power amplifier MMIC and the chip circulator, each circuit was fabricated on the Duroid 5,880 substrate (dielectric permittivity $=2.2 \pm 0.02$, dielectric thickness $=254 \mu \mathrm{m}$, metal thickness $=8 \mu \mathrm{m})$.

A voltage-controlled oscillator with a buffer amplifier generated an oscillation signal at $17.97 \mathrm{GHz}$ with source feedback topology. The output power was $19 \mathrm{dBm}$ (including connector loss) using bare-chip GaAs transistors of $4 \times 75 \mu \mathrm{m}$ and the result is shown in Fig. 3. The resolution and the video bandwidths are $100 \mathrm{kHz}$. The oscillation frequency can be varied with bias control from 17.7 to $18.2 \mathrm{GHz}$.

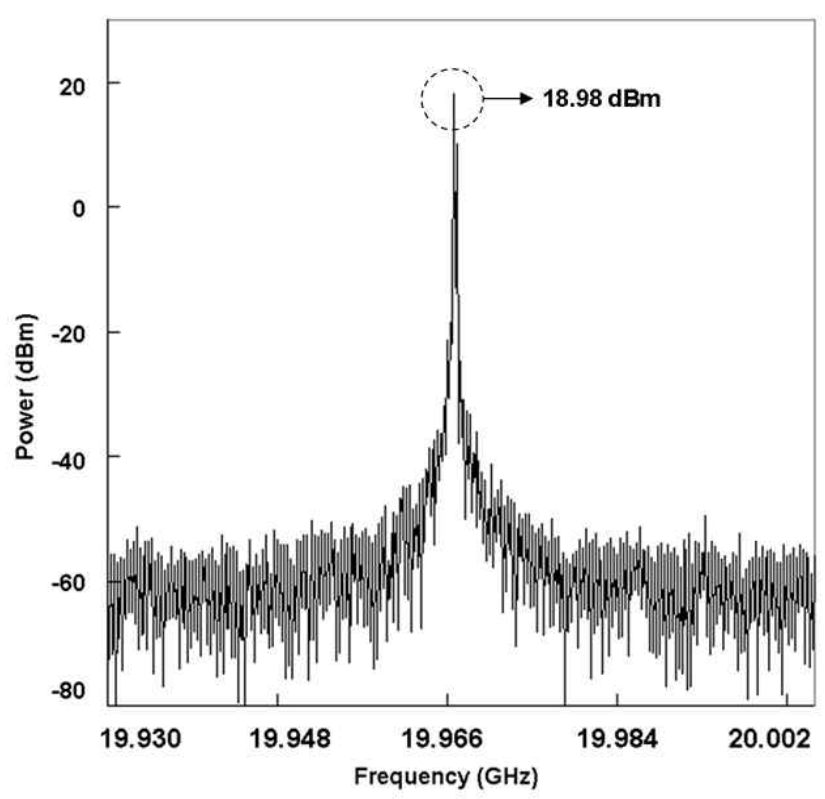

Fig. 3. The measured spectrum of the designed $18 \mathrm{GHz}$ VCO.

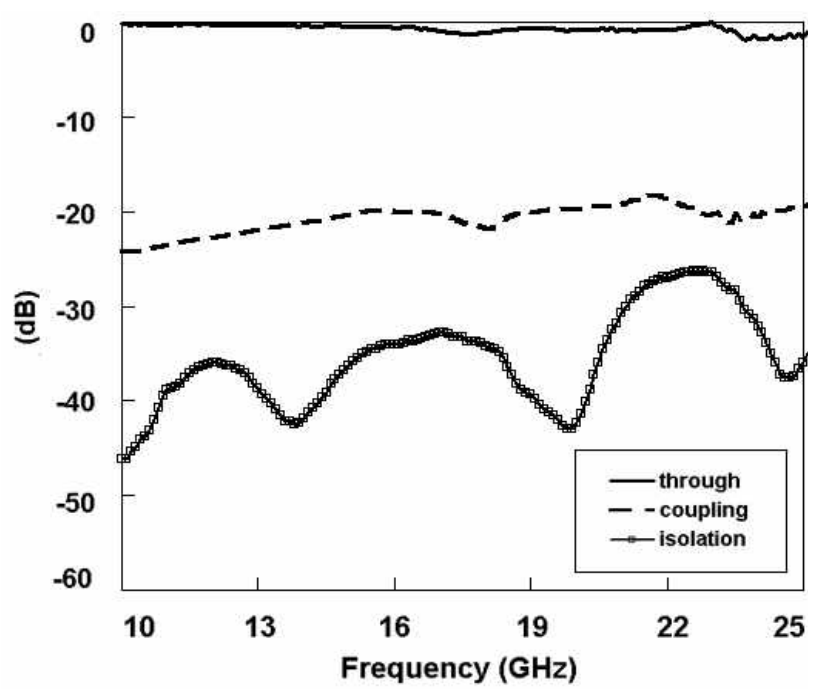

Fig. 4. Measured S-parameters of the designed coupledline coupler.

A coupled-line coupler was designed and fabricated to monitor the incident power of the hyperthermia system with a $20 \mathrm{~dB}$ coupling factor at the designed frequency of $18 \mathrm{GHz}$. The coupler was designed using two 50 $\operatorname{Ohm}(770 \mu \mathrm{m})$ microstrip lines with a gap of $100 \mu \mathrm{m}$ and an overlap length of $1,800 \mu \mathrm{m}$. The insertion loss (through), coupling and isolation of the coupler were $1.3,21.7,34.2 \mathrm{~dB}$, respectively(including connector loss). Fig. 4 shows measured S-parameters of the fabricated 20 dB coupled-line coupler.

A commercial microstrip circulator(RADITEK, RADC- 


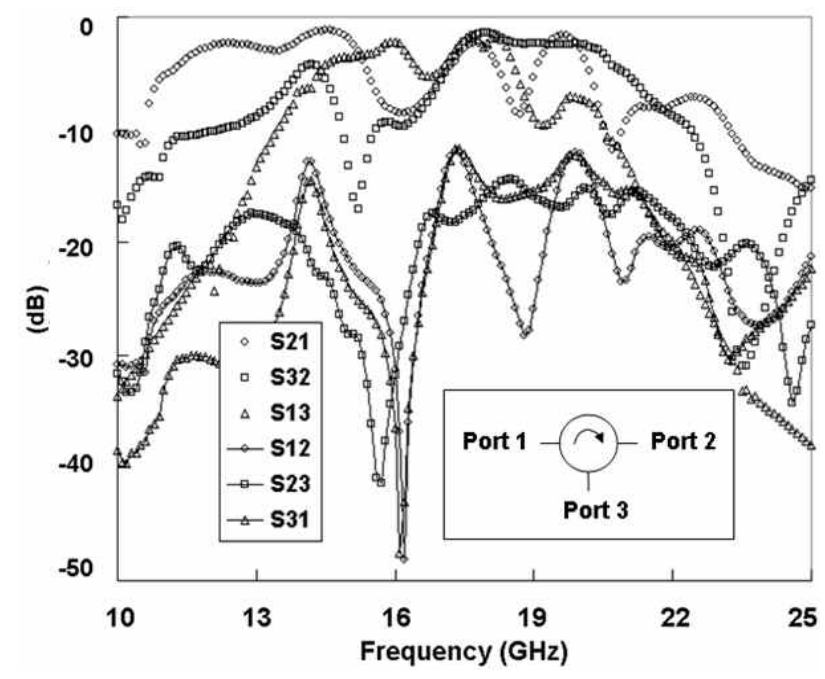

Fig. 5. Measured S-parameters of the circulator.

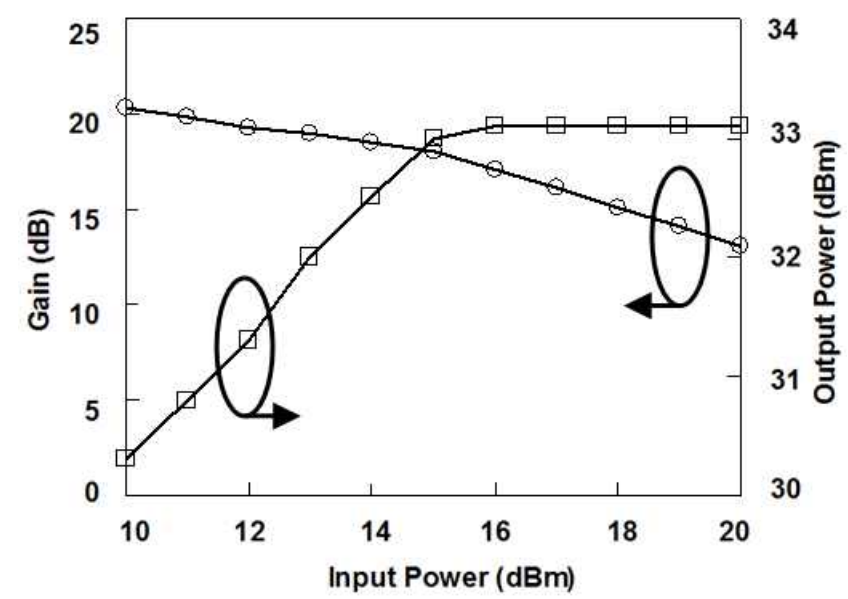

Fig. 6. The measured output power and the power gain of the amplifier.

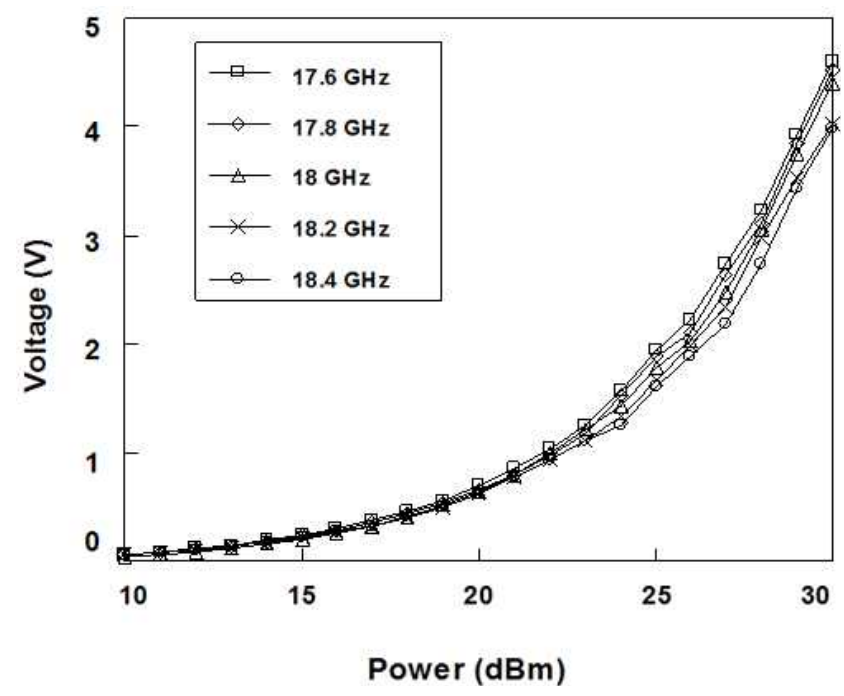

Fig. 7. Measured voltages of the power detector for various frequencies.

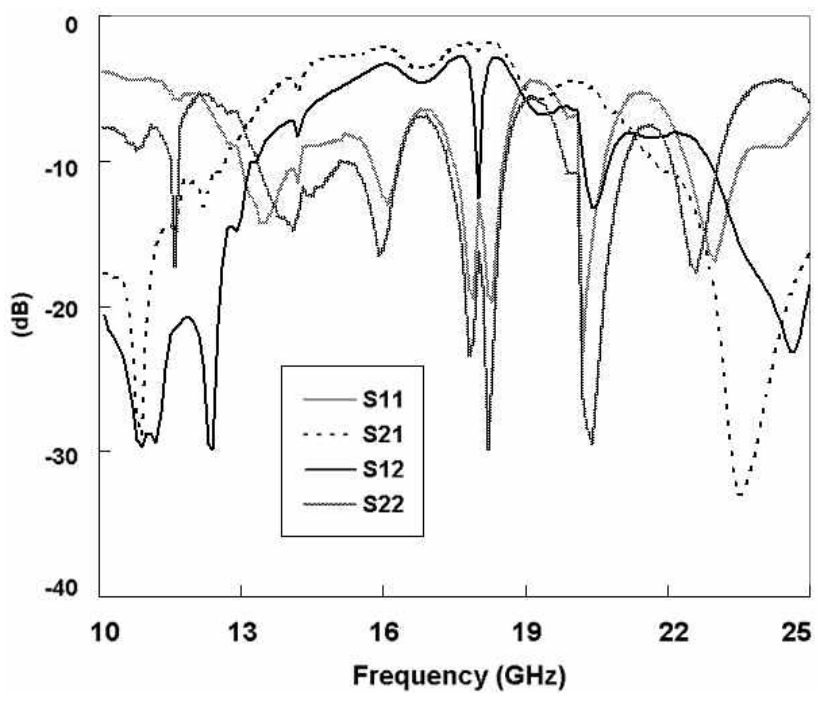

Fig. 8. Measured S-parameters of the second module.

17.2-18.7-MS31-2WR) was used to measure the reflected power with isolation of $20 \mathrm{~dB}$. The power from the signal source passes through a coupler and a circulator, the incident power can be monitored from a 20-dB coupled port of the coupler and the reflected power can be monitored from the circulator. Measured S-parameters of the circulator are shown in Fig. 5. The average values of the insertion loss and isolation are 1.6 and $20.7 \mathrm{~dB}$ (including connector loss) from 17.6 to 18.4 $\mathrm{GHz}$, respectively.

A commercial high-power and high-gain amplifier (TriQuint Semiconductor, TGA2514-FL) was used to amplify the signal from the VCO after a buffer amplifier. Since an output power of at least $1 \mathrm{~W}$ is needed to perform microwave hyperthermia, a high-power and high-gain amplifier is one of the most important components of the hyperthermia system. The saturated output power of the amplifier is $33.2 \mathrm{dBm}$ and the power gain is $13.4 \mathrm{~dB}$. Considering losses from signal paths, over $1 \mathrm{~W}$ of power can be emitted from the system output. The measured power and the gain of the power amplifier are shown in Fig. 6. Because the heat from the operating amplifier affects overall performances of the Power Amplifier Module and other circuits, a separate module block(Module 2 in Fig. 1) has been dedicated to PAM. Heat-sinking fin was attached to the power amplifier block.

A power detector was designed and fabricated with two bare-chip GaAs transistors of $2 \times 20 \mu \mathrm{m}$. The drain and sources of the transistors were combined with wire bonds to function as diodes. Fig. 7 shows measured voltages for various frequencies. As shown in Fig. 7 the bandwidth of the power detector is about $0.8 \mathrm{GHz}$. Fig. 8 shows measured S-parameters of Module 2(in Fig. 1). 


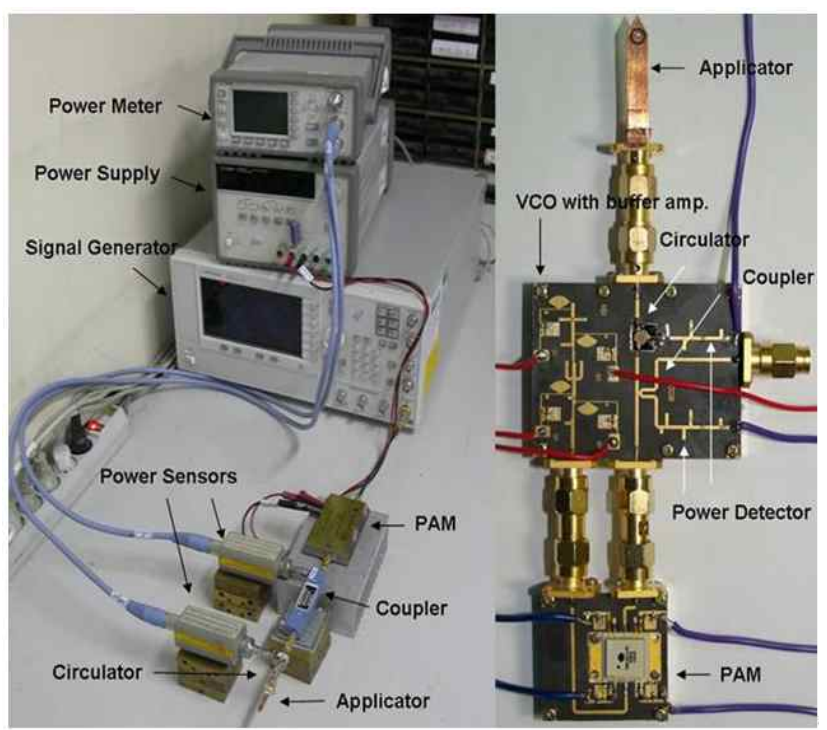

(a) Components-based systems

(b) Miniaturized system

Fig. 9. The components-based (a) and the miniaturized (b) microwave hyperthermia systems.

The insertion loss through a $20 \mathrm{~dB}$ coupler and a circulator is $1.9 \mathrm{~dB}$ at $18 \mathrm{GHz}$ and return losses are over $10 \mathrm{~dB}$ from 17.5 to $18.4 \mathrm{GHz}$. The total output power of the hyperthermia system was $1.35 \mathrm{~W}$ at 18 $\mathrm{GHz}$.

\section{Measurements}

Fig. 9 shows a photographic comparison of the components-based and the miniaturized microwave hyperthermia systems. In the miniaturized system, the size of the PAM is $3 \times 3 \mathrm{~cm}^{3}$ and that of the second module is $3 \times 5 \mathrm{~cm}^{3}$. The total size of the miniaturized system including two connectors is $10 \times 6.5 \mathrm{~cm}^{3}$ (excluding applicator). The planar coaxial applicator at the output of the miniaturized system was also fabricated on Duroid 5880 substrate, which was the same substrate used to build the hyperthermia system. The details of the planar coaxial applicator can be found in our previous work ${ }^{[8]}$. The loss of the applicator including connector loss was 1.2 $\mathrm{dB}$ at $18 \mathrm{GHz}$. Thus, the output power of the system including the applicator was $1.1 \mathrm{~W}$.

The hyperthermia system was tested on xenografted nude mice. Fig. 10 shows IR(Infra Red) images of a nude mouse with a cancer after 5 minutes of ablation. The first one is an IR image of a normal view with an inserted applicator on a nude mouse and the second one is a rescaled IR image of the first one over $43{ }^{\circ} \mathrm{C}$. The area of the surface of which the temperature was above $43{ }^{\circ} \mathrm{C}$ was defined as the "ablated area" of the surface ${ }^{[1]}$. The average value of the ablated area calculated from five experiments was $41 \%$ of the total area of a cancer.

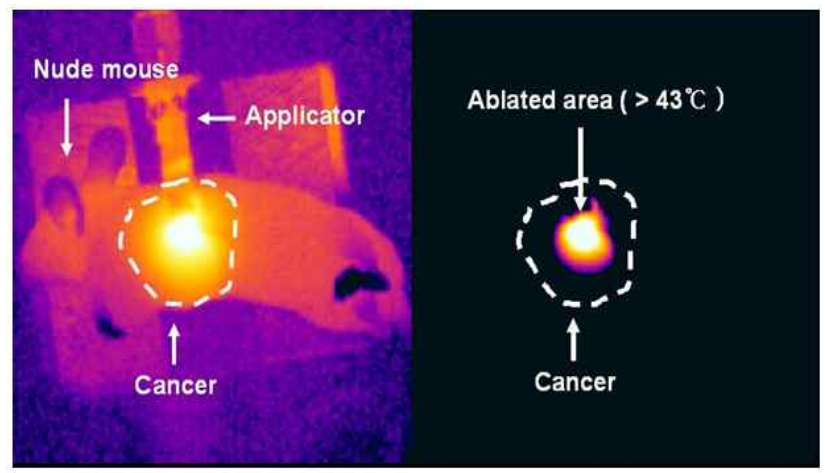

(a) An IR image of a normal view

(b) The rescaled(over $43{ }^{\circ} \mathrm{C}$ ) same IR image of(a)

Fig. 10. IR images of a normal view (a) and an over $43{ }^{\circ} \mathrm{C}$ view (b) after 5 minutes of a hyperthermia.

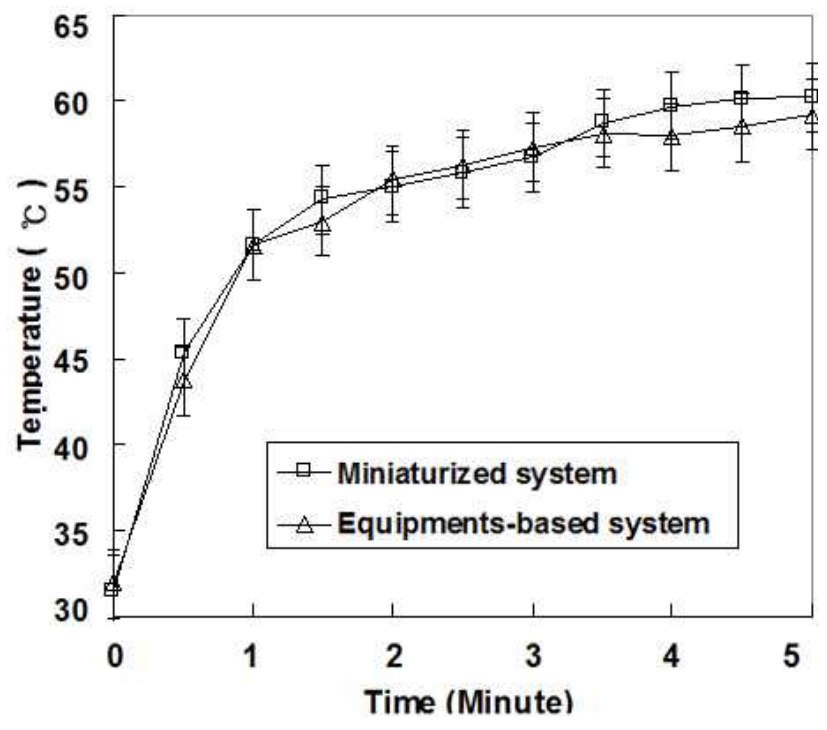

Fig. 11. Measured ablation results of cancer on nude mice with the components-based and the miniaturized systems.

The area can be broader as the power becomes higher.

Fig. 11 shows measured ablation results of nude mice with cancer, compared with those of the componentsbased system under the same experimental conditions with power of $1.1 \mathrm{~W}$ for 5 minutes. Each set of data consists of values averaged over five times. As shown in the figure, the temperature difference between the components-based and the miniaturized systems is within the error-bars. During the ablation, most of data are almost the same with a maximum temperature difference of $1.8{ }^{\circ} \mathrm{C}$ at 4 minutes $\left(59.7\right.$ and $57.9{ }^{\circ} \mathrm{C}$ in the components-based and the miniaturized systems, respectively). The above results indicate that the miniaturized microwave hyperthermia system can successfully replace the massive components-based one without performance degradation. 


\section{Conclusion}

A miniaturized hyperthermia system was developed using high-frequency microwaves. The system could be divided into two modules. The first one was a PAM (Power Amplifier Module) and the second one was made of integrated circuits consisting of a VCO with a buffer amplifier, a 20-dB-coupled line coupler, a chip circulator and two power detectors. As a signal source, a VCO integrated with a buffer amplifier generated an output power of $19 \mathrm{dBm}$ at $17.97 \mathrm{GHz}$. Following the VCO, a PAM having a $2 \mathrm{~W}$ of output power was employed. The 20-dB-coupled line coupler and a power detector with a bandwidth of over $1 \mathrm{GHz}$ were used to monitor the incident power. The circulator and the other power detector were also used to monitor the reflected power. The integrated modules had small insertion loss, maintaining over $1 \mathrm{~W}$ of output power for the entire system. In order to verify the miniaturized system, ablations were performed on nude mice xenografted with human breast cancer. From ablated results of both systems, it could be seen that the massive and expensive system could successfully be replaced by a small and low-cost planar system. This method can be a promising approach to implement a low-cost and practical microwave ablation system in clinical environments.

This work was supported by the Brain Korea 21 Program(BK21) and the Acceleration Research Program of the Ministry of Education, Science and Technology of the Republic of Korea and the Korea Science and Engineering Foundation.

\section{References}

[1] C. K. Chou, "Application of electromagnetic energy in cancer treatment", IEEE Trans. Instrumentation and Measurement, vol. 37, pp. 547-551, 1988.

[2] J. C. Lin, "Biomedical application of electromagnetic engineering", in Handbook of Engineering Electromagnetics, pp. 605-629, 2004.

[3] A. J. Fenn, A. Y. Cheung, and H. Cao, "Adaptive focusing experiments for minimally invasive monopole phased array in hyperthermia treatment of cancer", Proceedings of Engineering in Medicine and
Biology Conference of IEEE, vol. 2, pp. 766-767, Nov. 1994.

[4] J. A. Paulus, J. S. Richardson, R. D. Tucker, and J. B. Park, "Evaluation of inductivelyheated ferromagnetic alloy implants for therapeutic interstitial hyperthermia", IEEE Transactions on Biomedical Engineering, vol. 43(4), pp. 406-413, Apr. 1996.

[5] M. Hiraoka, M. Mitsumori, N. Hiroi, S. Ohno, Y. Tanaka, Y. Kotsuka, and K. Sugimachi, "Development of RF and microwave heating equipment and clinical applications to cancer treatment in Japan", IEEE Transactions on Microwave Theory and Techniques, vol. 48, no. 11, pp. 1789-1799, Nov. 2000.

[6] B. Emami, C. A. Perez, L. Leybovich, W. Straube, and D. Vongerichten, "Interstitial thermoradiotherapy in treatment of malignant tumors", International Journal of Cancer, vol. 3, no. 2, pp. 107-118, 1987.

[7] B. Prevost, S. D. Cordoue, X. Mirabel, J. C. Camart, J. J. Fabre, J. P. Sozanski, and M. Chive, "915 $\mathrm{MHz}$ microwave interstitial hyperthermia. part III: phase II clinical results", International Journal of Cancer, vol. 9, no. 3, pp. 455-462, 1993.

[9] K. Saito, H. Yoshimura, K. Ito, Y. Aoyagi and H. Horita, "Clinical trials of interstitial microwave hyperthermia by use of coaxial-slot antenna with two slots", IEEE Transactions on Microwave Theory and Techniques, vol. 52(8), pp. 1987-1991, Aug. 2004.

[10] W. Hurter, F. Reinbold, and W. J. Lorenz, "A dipole antenna for interstitial microwave hyperthermia", IEEE Transactions on Microwave Theory and Techniques, vol. 39(6), pp. 1048-1054, Jun. 1991.

[11] J. Cho, J. Yoon, S. Cho, K. Kwon, S. Lim, D. Kim, E. Lee, C. Kim, J. Choi, C. Cheon, and Y. Kwon, "In-vivo measurements of the dielectric properties of breast cancer carcinoma xenografted on nude mice", International Journal of Cancer, vol. 119(3), pp. 593-598, Mar. 2006.

[12] B. Kang, J. Park, J. Cho, K. Kwon, S. Lim, J. Yoon, C. Cheon, Y. Kim, and Y. Kwon, "Novel low-cost planar probes with broadside apertures for nondestructive dielectric measurement of biological materials at microwave frequencies", IEEE Transactions on Microwave Theory and Techniques, vol. 53(1), pp. 361-371, Jan. 2005. 


\section{Dongki Kim}

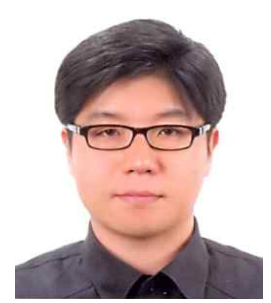

thermia system. received the B.S. degree in electrical engineering from Sung Kyun Kwan University, in 2001, the M.S. degree in electrical engineering from Seoul National University, in 2003, and is currently working toward the Ph.D. degree at Seoul National University. His research is focused on the design of applicator and microwave hyper-

\section{Kihyun Kim}

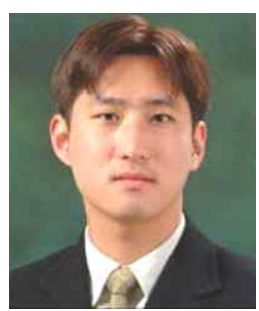

received the B.S. and M.S. degrees from Seoul National University, in 2003 and 2008, respectively, and is currently working toward the Ph.D. degree at the Seoul National University. His research interests are the measurement of complex permittivity and the design of microwave systems for cancer detection

\section{Jungmin $\mathrm{Oh}$}

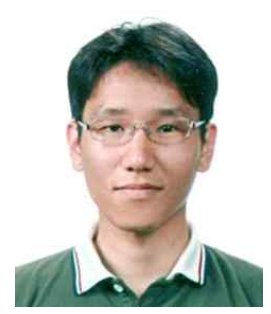

received the B.S. degree in school of information and communication engineering from Sung Kyun Kwan University in 2006, the M.S. degree from Seoul National University in 2009 , and is currently working in AVAGO technologies.

\section{Youngrak Park}

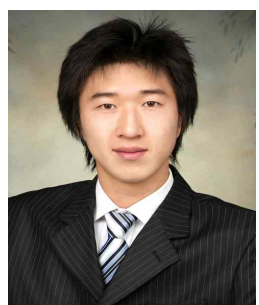

received the B.S. degree in electronic engineering from Information and Communication University, and is currently working toward the M.S. degree in Seoul National University. His research interests is the design of power amplifier and microwave circuits.

\section{Youngwoo Kwon}

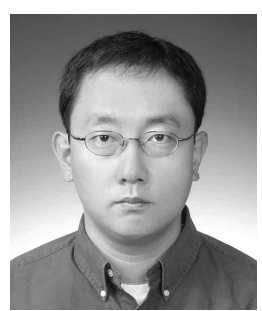

recieved the B.S. degree in electronics engineering from Seoul National University in 1988, and the M.S. and Ph.D. degrees in electrical engineering from the University of Michigan, Ann Arbor, in 1990 and 1994, respectively. Professor Kwon is currently a senior member of the IEEE, and has been working as an Associate Editor for IEEE Transactions on Microwave Theory and Techniques. He has authored and coauthored over 150 technical papers in the internationally renowned journals and conferences. He was the recipient of Presidential Young Investigator award from Korean government in 2006. 